\title{
RELAÇÃO ENTRE O INVESTIMENTO SOCIAL CORPORATIVO E O VALOR DAS EMPRESAS BRASILEIRAS
}

\section{RELATIONSHIP BETWEEN CORPORATE SOCIAL INVESTMENT AND THE VALUE OF BRAZILIAN COMPANIES}

\section{RELACIÓN ENTRE LA INVERSIÓN SOCIAL CORPORATIVA Y EL VALOR DE LAS EMPRESAS BRASILEÑAS}

\section{ROGIMÁRIO MENEZES DE OLIVEIRA}

Mestre em Contabilidade pela Fucape Business School

rogimario@bol.com.br

ANNOR DA SILVA JUNIOR

Doutor em Administração pela UFMG

annorsj@fucape.br

\section{ALFREDO RODRIGUES LEITE DA SILVA}

Doutor em Administração pela UFMG

alfredosilva@fucape.br

\section{RESUMO}

Este estudo tem por objetivo investigar a existência de relação entre os investimentos sociais corporativos e o valor das empresas listadas na Bolsa de Valores de São Paulo (Bovespa) e que publicam balanço social no Instituto Brasileiro de Análises Sociais e Econômicas (Ibase), mais especificamente, identificando se empresas que investem sistematicamente em responsabilidade social têm seu valor potencializado ou diminuído. Os resultados encontrados não evidenciaram que investimentos em práticas de responsabilidade social acarretam um melhor ou pior desempenho no valor de mercado das empresas. Encontrou-se relação não significativa 
entre a variável de controle tamanho da empresa e o q de Tobin em empresas com investimentos sociais corporativos. Encontrou-se relação negativa entre alguns setores de mercado e o valor das empresas representados pelo q de Tobin. Foi confirmado por esta pesquisa a relação positiva entre o tamanho das empresas e o valor dos investimentos sociais corporativos.

Palavras-chave: Responsabilidade Social, Contabilidade, desempenho.

\section{ABSTRACT}

This study aims to investigate the existence of the relationship between corporate social investments and value of companies listed on the São Paulo Stock Exchange (Bovespa) and which publish their social balance sheets in the Brazilian Institute of Social and Economic Analyses (IBASE), more specifically, identifying if companies that systematically invest in social responsibility have their value increased or reduced. The results found did no evidence that investments in social responsibility practices imply better or worse performance in the market value of companies. It was found a non-significant relationship between the control variable size of the company and the Tobin's $q$ in companies with corporate social investment. It was found a negative relationship between some sectors of the market and the value of the companies represented by Tobin's $q$. It was confirmed by this research the positive relationship between companies' size and value of corporate social investment.

Keywords: Social Responsibility, Accounting, Performance.

\section{RESUMEN}

Este estudio tiene por objetivo investigar la existencia de relación entre las inversiones sociales corporativas y el valor de las empresas listadas en la Bolsa de Valores de São Paulo (Bovespa) y que publican balance social en el Instituto Brasileño de Análisis Sociales y Económicos (Ibas) más específicamente, identificándose empresas que invierten sistemáticamente en responsabilidad social tiene su valor potencializado o disminuido. Los resultados encontrados no evidenciaron que inversiones en prácticas de responsabilidad social acarrean un mejor o peor desempeño en el valor de mercado de las empresas. Se encontró relación no significativa entre la variable de control de tamaño de la empresa y la q de Tobin en empresas con inversiones sociales corporativas. Se encontró relación negativa entre algunos sectores de mercado y el valor de las empresas representados por la $\mathrm{q}$ de Tobin. Fue confirmada por esta investigación la relación positiva entre el tamaño de las empresas y el valor de las inversiones sociales corporativas.

Palabras clave: Responsabilidad Social, Contabilidad, desempeño. 


\section{repec}

\section{INTRODUÇÃO}

A partir dos anos 50, nos Estados Unidos e na Europa, já se verificava uma mutação nos valores da conjuntura socioeconômica, gerando reivindicações generalizadas por parte da sociedade em relação ao contexto socioambiental das empresas e sua forma de atuação. Nesse novo contexto, a Teoria dos Stakeholders, ou teoria das partes relacionadas, surge como alternativa conceitual administrativa, que ressalta os objetivos estratégicos de se atender ao interesse das "partes interessadas" que afetam ou são afetados pelos objetivos da empresa (FREEMAN, 1984). A Teoria dos Stakeholders vêm sendo objeto de estudo dos mais diversos autores, sendo caracterizada por três aspectos por Donaldson e Preston (1995, p. 66 e 67): descritiva, instrumental e normativa. É o aspecto instrumental, a teoria utilizada para identificar conexões (ou a ausência delas) entre a administração dos Stakeholders e os objetivos de desempenho corporativo (DONALDSON; PRESTON, 1995, P. 71). Por sua vez, a responsabilidade social corporativa está alinhada e inserida no conceito da Teoria dos Stakeholders, sendo definida como expectativas econômicas, éticas, legais e discricionárias que a sociedade possui em relação às organizações em determinado período de tempo (CARROL, 1979, P.500). Ao tornar-se socialmente responsável, as organizações acabam por constituir uma ampla gama de relações, envolvendo diversos atores sociais que possuem interesses distintos, que são chamados de Stakeholders. Dentre esses, destacam-se: acionistas e investidores; agentes financeiros; pessoas físicas e jurídicas contratadas para trabalhar com a empresa (empregos diretos e terceirizações); concorrentes; parceiros privados, estatais e entidades não governamentais; cadeia de compradores/consumidores (diretos e indiretos); cadeia de fornecedores (diretos e indiretos); ambiente local, regional e mundial; a comunidade local; os governos federal, estadual e municipal; gerações futuras de Stakeholders, etc.

Vários trabalhos acadêmicos têm enfocado a importância da responsabilidade social corporativa no âmbito do conceito da Teoria dos Stakeholders. Algumas organizações que se omitiram em agir responsavelmente com seus Stakeholders sofreram deterioração no preço de suas ações e lucros (FERREL et al., 2001). Cornell e Shapiro (1987) sustentaram a teoria de que existe associação positiva entre o desempenho da empresa com responsabilidade social, correlacionando a relação de causa e efeito com a Teoria dos Stakeholders. Para Preston e O`Bannon (1997, p.420), a associação positiva entre desempenho social e financeiro corporativo seria uma implicação crítica em favor da Teoria dos Stakeholders. McGuirre et al. (1988) analisaram a relação entre a responsabilidade social das empresas e medidas de desempenho financeiro, por meio de indicadores contábeis, encontrando uma correlação significativa de forma positiva. Bromiley e Marcus (1989) encontraram relação negativa entre esses dois desempenhos. Ruf et al. (2001) investigaram a relação entre desempenho social econômico e concluíram que as mudanças no desempenho social estão positivamente associadas com o crescimento dos receitas (vendas), prevalecendo ambí- 
guas até o momento as conclusões dos trabalhos científicos sobre este tema. Externalidades, segundo Pindyck e Rubinfeld (2002, p.631), se constituem em importantes causas de falhas de mercado, dando origem a sérias questões relacionadas com o investimento social corporativo. Tais autores assim conceituaram "externalidades":

Há externalidades negativas - que ocorrem quando a ação de uma das partes impõe custos à outra - e externalidade positivas - que surgem quando a ação de uma das partes beneficia a outra. (PINDYCK e RUBINFELD, 2002, p. 631).

Um exemplo de externalidade negativa é quando empresas lançam efluentes em um rio próximo de uma área onde empresas de pesca estão situadas. Quanto mais efluentes lançados no rio, menos peixes serão pescados pelos barcos pesqueiros. Ou seja, quanto maior for a produtividade dessas empresas (que lançaram mais efluentes no rio), maiores serão os prejuízos que imporão às empresas pesqueiras.

Assim, à medida que o nível de produção das empresas poluidoras varia, o custo externo que recai sobre as empresas de pesca também varia. Foi inicialmente abordado pelo economista Ronald Coast, no seu artigo publicado em 1960, "The problem of Social Cost" (O problema do Custo Social), tornando-se famoso por relacionar o conceito de externalidades com os direitos de propriedade, como forma de gerar um resultado eficiente entre os agentes.

\section{Resultados de trabalhos anteriores}

O conflito acadêmico nessa área tem sido intenso para buscar respostas se as empresas socialmente responsáveis eram também empresas lucrativas. Três trabalhos clássicos que revisaram um grande número de pesquisa nessa área foram os trabalhos de Ullmann (1984) e Griffin e Mahon (1997) e o de Preston e O`Bannon (1997).

As relações entre ações corporativas de responsabilidade social e performance econômica foram analisadas no estudo de Ullmann (1985), que revisou 14 trabalhos de diferentes autores desde o ano de 1965 até 1975, sendo que 7 apresentaram correlação positiva, 5 estudos apresentaram correlação negativa e 2 estudos foram inconclusivos.

Ao analisar a relação entre a responsabilidade social das empresas e medidas de desempenho financeiro avaliados por indicadores contábeis, esses eram correlacionados de forma positiva com responsabilidade social.

Griffin e Mahon (1997, p. 6) realizaram uma revisão dos principais trabalhos durante 25 anos de pesquisas sobre o assunto. No total, 51 estudos, compreendendo o período de 1970 a 1994, foram revisados buscando um melhor entendimento entre o relacionamento entre ações socialmente responsável e performance financeira. Os resultados encontrados foram os seguintes: a) na década de 1970: em um total de 16 estudos, 12 apresentaram correlação positiva, 1 apresentou correlação negativa, 3 foram inconclusivos; b) na década 
de 1980: em um total de 27 estudos, 14 apresentaram correlação positiva, 11 apresentaram correlação negativa, 2 foram inconclusivos; c) na década de 1990: em um total de 8 estudos, 5 estudos encontraram relação positiva; e em 3 estudos a relação foi negativa.

Preston e O`Bannon (1997) realizaram um estudo de onze anos e encontraram resultados positivos para a hipótese de que maior nível de desempenho financeiro acarreta maior nível de desempenho social. A pesquisa foi realizada em 67 companhias por um período de 11 anos, entre 1982 e 1992. O pesquisador conclui o seguinte, após empregar ampla base de dados:

"encontramos fortes evidências de uma positiva relação entre ações sociais e performance financeira em indicadores de uma amostra de grandes e importantes corporações americanas encontrando ampla consistência com a teoria dos stakeholders da firma” (PRESTON e O`BANNON, p. 48, 1997).

Uma pesquisa realizada no Brasil, que serviu de referência para o presente trabalho, foi a de Borba (2005), que utilizou dados do Balanço Social no modelo do Instituto Brasileiro de Análises Sociais e Econômicas (Ibase) das empresas listadas na Bovespa para construir o indicador de desempenho social; e, para construir o indicador de desempenho financeiro corporativo, utilizou o $q$ de Tobin como medida de valor de mercado da empresa. O autor assim concluiu sua pesquisa:

"De maneira geral, os resultados encontrados por esta dissertação não corroboraram de maneira incisiva para a hipótese alternativa de "Impacto Social", de, ou seja, de que um melhor ou pior desempenho social corporativo acarretaria um melhor ou pior desempenho financeiro respectivamente. Dessa forma, as vantagens estratégicas da adoção de uma administração dos stakeholders voltada para o interesses de todos os envolvidos direta ou indiretamente em suas atividades não foram evidenciados na amostra e período considerados na pesquisa."(BORBA, 2005, p. 115).

Um aspecto significativo para as pesquisas acadêmicas relacionado à construção de modelos é a influência do tamanho da empresa no resultado dos testes empíricos como variável explicativa para o desempenho social e financeiro da empresa. É com base nesse quadro que se estabelece o problema de pesquisa aqui apresentado:

Qual a relação entre o investimento social corporativo e o valor das empresas brasileiras com ações negociadas na Bovespa que publicam regularmente o balanço social no lbase?

Definiu-se como objetivo geral identificar a relação entre o investimento social corporativo e o valor das empresas brasileiras com ações negociadas na Bovespa com o balanço social do Ibase. Como objetivos específicos, foram relacionados: 
- Identificar o rol de empresas de capital aberto com ações na Bovespa que publicaram balanço social no modelo do Instituto Brasileiro de Análises Sociais e Econômicas (Ibase) nos anos de 2002, 2003, 2004.

- Levantar dados dos balanços contábeis e do mercado de ações das empresas de capital aberto com ações na Bovespa nos anos de 2002, 2003 e 2004.

- Calcular e analisar o valor da empresas ( $q$ de Tobin) que fazem parte da amostra.

- Calcular e analisar o valor investido em práticas de responsabilidade social, seguindo o modelo do Instituto Brasileiro de Análises Sociais e Econômicas (Ibase).

- Analisar comparativamente os investimentos sociais listados no balanço social com o valor da empresa ( $q$ de Tobin).

Na operacionalização dos objetivos geral e específicos, elaboraram-se duas hipóteses:

H1: Existe relação entre os investimentos sociais corporativos e o valor da empresas brasileiras com ações negociadas na Bovespa.

H2: Os investimentos sociais corporativos relacionam-se positivamente com o valor das empresas brasileiras com ações negociadas na Bovespa.

Tendo em vista que a teoria enfatiza que o tamanho das empresas e o setor de atuação delas influenciam no valor dos investimentos sociais corporativos, a fim de buscar o reflexo do investimento social, o tamanho e o setor, no valor das empresas, foram elaboradas as seguintes hipóteses complementares:

H3: Existe uma correlação positiva entre o tamanho e o valor das empresas brasileiras com investimentos sociais e ações negociadas na Bovespa.

H4: Existe uma correlação positiva entre o setor e valor das empresas brasileiras com investimentos sociais e ações negociadas na Bovespa.

Limitações devem ser consideradas na pesquisa: primeiro, a utilização de apenas dados secundários. Apesar de serem companhias abertas e registradas na Bovespa e fiscalizadas pelos órgãos reguladores e por auditorias externas, não elimina a possibilidade de erros na medida e na manipulação dos números das companhias, que podem gerar informações inadequadas para os resultados obtidos pelo modelo da pesquisa.

\section{MÉTODO ADOTADO}

Metodologicamente, a pesquisa configura-se como quantitativo-descritivo do tipo estudo de verificação de hipóteses. Dessa forma, as hipóteses explícitas derivadas da teoria são verificadas por meio e um modelo estatístico de análise (TRIPODI et al., 1975). 
Utilizaram-se dados secundários disponíveis no banco de dados da Economática, em balanços e demonstrações financeiras de empresas listadas na Bovespa e que publicam balanços sociais publicados pelo Instituto Brasileiro de Análises Sociais e Econômicas (lbase).

Para a determinação do valor da empresa, utilizou-se como proxy o q de Tobin para os anos de 2002, 2003 e 2004. Para a determinação dos valores investidos em responsabilidade social, utilizou-se a soma total dos indicadores sociais internos, dos indicadores sociais externos e investimentos em meio ambiente.

O tamanho da amostra que atendia aos requisitos básicos da pesquisa (ações negociadas na Bovespa que publicam balanço social no lbase) foi de 43 empresas para o ano de 2002; 52 empresas para o ano de 2003; e 42 empresas para o ano de 2004.

\section{Modelo de Análise}

Log $q$ Tobin $=\&+B 1($ LogINVESTS $)+B 2($ LogTAM $)+B 3($ DIND1 $)+\ldots . .+B 13($ DIND10 $)+$ $B 14($ T2003 $)+B 15($ T2002 $)+B($ Log INVEST. DIND1 $)+\ldots . .+B($ LogINVEST. DIND10 $)+E$

Quadro 1 - Quadro de discriminação das variáveis

\begin{tabular}{|c|c|}
\hline Variável Dependente & Proxy \\
\hline (Valor de Mercado empresa) - $q$ de Tobin & $\begin{array}{l}q \text { de Tobin }=(\text { VMAQ +VMAP +DIVT) / AT } \\
\text { VMAQ - valor de mercado das ações ordinárias } \\
\text { VMAP - valor de mercado das ações preferenciais } \\
\text { DIVT - valor contábil das dividas de curto prazo somado ao valor } \\
\quad \text { contábil das dividas de longo prazo meno o ativo circulante } \\
\quad \text { + o estoque } \\
\text { AT - ativo total da campanhia. }\end{array}$ \\
\hline \multicolumn{2}{|l|}{ Variáveis Independentes } \\
\hline $\begin{array}{l}\text { LogINVESTS: Logaritmo do total do } \\
\text { Investimento em responsabilidade so- } \\
\text { cial da empresa }\end{array}$ & $\begin{array}{l}=\text { valor total dos indicadores sociais internos }+ \\
\text { valor total dos indicadores externos }+ \\
\text { valor total dos indicadores ambientais do balanço social (modelo lbase) }\end{array}$ \\
\hline $\begin{array}{l}\text { LogTAM (Logaritmo do total do tama- } \\
\text { nho da empresa) }\end{array}$ & $\begin{aligned} \text { Tamanho: }= & \text { faturamento líquido operacional } \\
& \text { valor em } 31 / 12 \text { dos anos de 2002, } 2003 \text { e } 2004 \\
& \text { do banco de dados Economática }\end{aligned}$ \\
\hline DIND (variável dummy da indústria) & $\begin{array}{l}\text { Indústria = } 20 \text { setores empresas capital aberto } \\
\text { da Bovespa descritos no banco de dados } \\
\text { Economática, exceto bancos e fundos }\end{array}$ \\
\hline
\end{tabular}

O INVESTS é o indicador de investimentos sociais corporativos desenvolvido a partir dos fundamentos teóricos do modelo de performance social corporativa proposto por Wood e Jones (1996), particularmente em relação aos efeitos para com os stakeholders internos e os efeitos para com os stakeholders externos. Esses stakeholders internos estão representados nos itens dos grupos dos indicadores sociais internos abaixo elencados, enquanto 
os stakeholders externos estão representados no grupo dos indicadores sociais externos e nos investimentos em meio ambiente. Para a composição do INVESTS, definiram-se: o valor da soma do "total dos indicadores sociais internos", do "total dos indicadores sociais externos" e do "total dos investimentos em meio ambiente".

Esses valores econômico-financeiros estão elencados nos balanços sociais publicados pela empresas brasileiras, conforme o quadro a seguir:

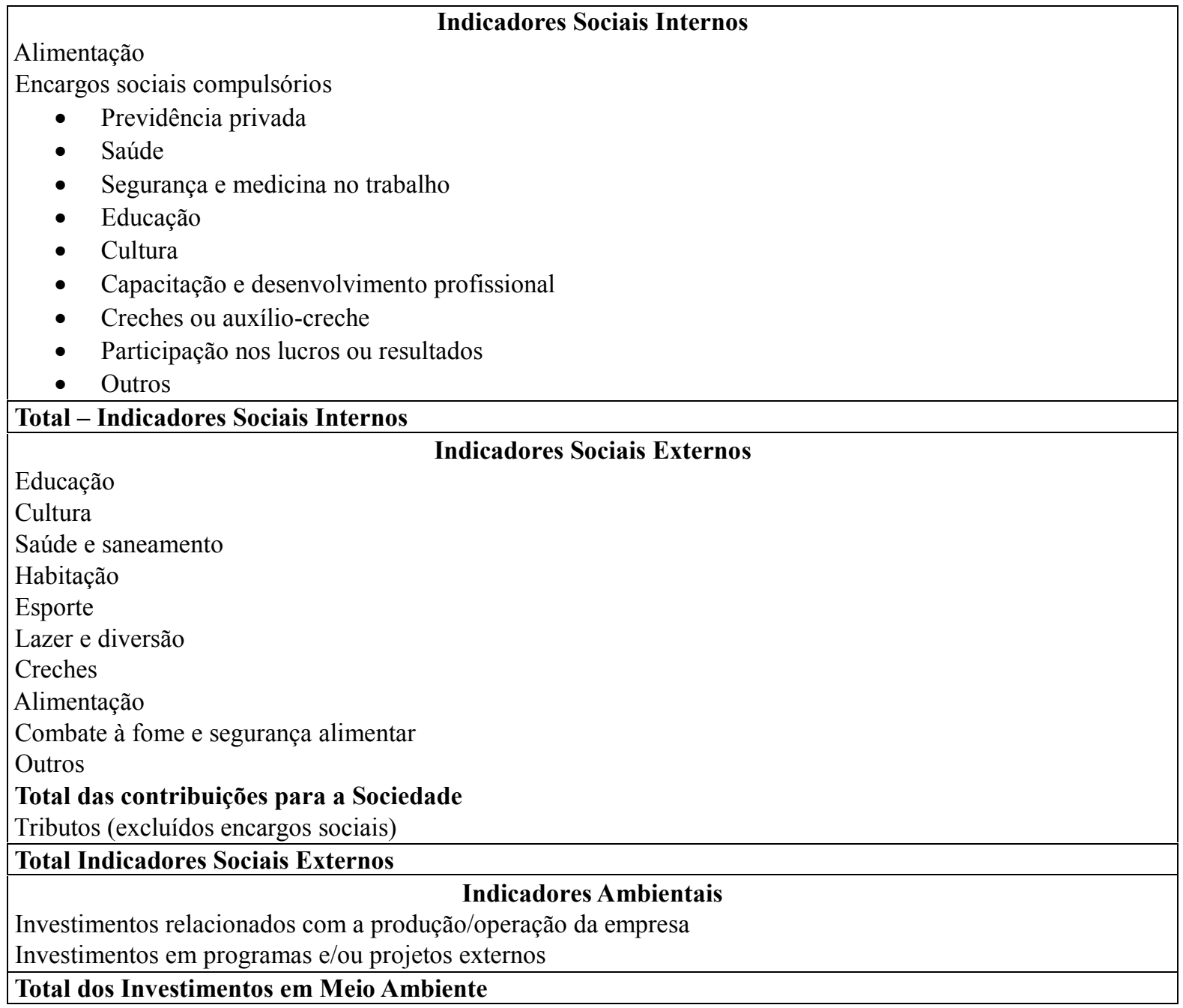

Fonte: Adaptado do balanço social do Ibase (2006)

Quadro 2: Indicadores de investimento social corporativo (INVESTS)

O LogINVESTS é a opção por inseri-la em escala logarítmica uma vez que o modelo de regressão pressupõe variáveis que exigem uma adequação estatística mais apropriada em relação a aspectos de assimetria e curtose.

As variáveis de controle consideradas no trabalho são o tamanho da empresa (TAM) e o setor de atuação (IND), já que são variáveis que podem exercer influência sobre a relação entre o investimento social corporativo e o desempenho da empresa (GRIFFIN; MAHON, 1997). 


\section{ANÁLISE DOS RESULTADOS}

Para a análise dos resultados deste trabalho, seguiu-se a seguinte ordem:

a) inicialmente é realizada uma análise descritiva dos dados para os anos de 2002, 2003 e 2004, destacando o resultado de cada período;

b) em seguida, é apresentada a matriz de correlações seguida de uma análise dos resultados para cada período;

c) posteriormente, é apresentado o resultado da regressão em pooled cross-section pelo método dos Mínimos Quadrados Ordinários (MQO), sendo analisados os seus coeficientes estatisticamente significantes ao nível de $5 \%$, verificando os pressupostos exigidos para o método regressivo, com erros padrões robustos à heterocedasticidade e confirmado um padrão de normalidade das amostras pelo teste de Kolmogorov-Smirnov, que pode ser aplicado para testar se a característica estudada da amostra é oriunda de uma população com distribuição normal.

\subsection{Análise Estatística Descritiva - 2002}

Tabela 1 - Estatística descritiva com valores originais 2002

\begin{tabular}{|l|c|c|c|c|c|c|}
\hline \multicolumn{1}{|c|}{ Variável } & N & Mínimo & Média & Máximo & Assimetria & Curtose \\
\hline$q$ de Tobin & 43 & 0,3536 & 0,8047 & 2,2025 & 1,30 & 1,68 \\
\hline Invests & 43 & 12856 & 325098 & 44650737 & 6,32 & 40,84 \\
\hline TAM & 43 & 439538 & 1747863 & 69176155 & 5,06 & 28,54 \\
\hline
\end{tabular}

Tabela 2 - Estatística descritiva em escala logarítmica 2002

\begin{tabular}{|l|c|c|c|c|c|c|}
\hline \multicolumn{1}{|c|}{ Variável } & N & Mínimo & Média & Máximo & Assimetria & Curtose \\
\hline $\log q$ de Tobin & 43 & 0,4514 & $-0,0944$ & 0,3429 & 0,27 & 0,08 \\
\hline $\log$ Invests & 43 & 4,1091 & 5,5120 & 7,6498 & 56 & 1,73 \\
\hline $\log$ TAM & 43 & 5,6430 & 6,2425 & 7,8400 & 0,97 & 0,89 \\
\hline
\end{tabular}

A estatística descritiva dos dados coletados para o ano de 2002 apresenta algumas informações importantes. Primeiramente, observa-se que, em escala logarítmica, os dados apresentaram um padrão estatístico de assimetria e curtose bem mais adequado para análise do que no formato original, conforme pode ser observado entre as Tabelas 1 e 2 .

O valor de mercado das empresas (log $q$ de Tobin -0,94) apresentou um padrão médio de maior proximidade do valor mínimo $(-0,45)$ do que do valor máximo $(0,34)$, indicando que a maioria das que publicaram o balanço social na Bovespa apresentaram, na média, um valor de mercado mais próximo do valor mínimo na população analisada em 2002.

A variável de controle de tamanho da empresa apresentou média mais elevada $(6,24)$ e mais centralizada em relação ao mínimo de 5,64 e máximo de 7,84. O resultado 
pode ser explicado pela maior presença na amostra de empresas de setores com elevado faturamento no ano 2002.

\subsection{Análise da Matriz de Correlações - 2002}

Tabela 3 - Matriz de correlações do ano de 2002

\begin{tabular}{|l|c|c|c|}
\hline \multicolumn{1}{|c|}{ Variável } & q de Tobin & Log INVTS & Log TAM \\
\hline $\log q$ de Tobin & 1,000 & & \\
\hline $\log$ INVTS & $-0,025$ & 1,000 & \\
\hline $\log$ TAM & 0,228 & 0,724 & 1,000 \\
\hline
\end{tabular}

As correlações diretas entre o investimento em responsabilidade social (INVESTS) e o valor da empresa ( $q$ de Tobin) mostraram-se fracas $(-0,025)$, não apresentando correlação estatística significativa.

A variável de controle tamanho apresentou correlação significante com a variável de investimento em responsabilidade social (log INVESTS) $(0,724)$.

Quanto ao sentido que as variáveis indicam:

1 A Correlação negativa encontrada entre o $q$ de Tobin e a variável INVESTS indica uma relação negativa entre o investimento em responsabilidade social corporativa e o valor das empresas.

2 A correlação, entretanto, não é significativa para se afirmar que, quanto maior o valor de mercado da empresa menor o investimento social corporativo, com base em valores da ordem de $-0,025$.

3 A Correlação positiva encontrada entre o log TAM e o log INVESTS indica uma relação entre o tamanho da empresa e o valor do investimento em responsabilidade social corporativa, indicando que quanto maior o tamanho da empresa maior o investimento em responsabilidade social com base em valores da ordem de 0,724. Isso pode significar que empresas com maior faturamento líquido também apresentam maior investimento social corporativo.

\subsection{Análise da Estatística Descritiva - 2003}

Tabela 4 - Estatística descritiva com valores originais 2003

\begin{tabular}{|l|c|c|c|c|c|c|}
\hline \multicolumn{1}{|c|}{ Variável } & N & Mínimo & Média & Máximo & Assimetria & Curtose \\
\hline $\log q$ de Tobin & 52 & 0,3336 & 1,0117 & 2,9435 & 1,18 & 1,27 \\
\hline $\log$ Invests & 52 & 32295 & 453385 & 48921005 & 6,80 & 47,85 \\
\hline TAM & 52 & 275241 & 219707 & 95742702 & 5,98 & 39,07 \\
\hline
\end{tabular}


Tabela 5 - Estatística descritiva utilizando valores em escala logarítmica

\begin{tabular}{|l|c|c|c|c|c|c|}
\hline $\begin{array}{c}\text { Variáveis } \\
\text { Independentes }\end{array}$ & $\mathbf{N}$ & Mínimo & Média & Máximo & Assimetria & Curtose \\
\hline log q de Tobin & 52 & 0,4768 & 0,00503 & 0,4689 & 0,09 & $-0,23$ \\
\hline log Invests & 52 & 4,5091 & 5,6565 & 7,6895 & 0,60 & 1,09 \\
\hline TAM & 52 & 5,4397 & 6,3410 & 7,9811 & 0,63 & 0,95 \\
\hline
\end{tabular}

A estatística descritiva dos dados coletados para o ano de 2003 apresentou um valor médio do investimento social (log INVESTS -5,65) maior que em 2002 (log INVESTS: 5,51). Embora ainda pequeno, revela um provável aumento na atuação social de algumas empresas ou maior transparência na divulgação dos dados do Balanço Social. Esse fato pode ser também observado nos valores de mínimo e de máximo que aumentaram em relação ao ano 2002.

Observou-se que, em escala logarítmica, os dados apresentaram um padrão estatístico de assimetria e curtose mais adequado para análise estatística.

A média de valor de mercado das empresas teve também um aumento em 2003 (log $q$ de Tobin 0,00503) em relação a 2002 (log $q$ de Tobin -0,0944). Esse aumento do valor médio de mercado também pode ser observado no distanciamento do valor médio do valor mínimo, estando mais ao centro, indicando que a maioria das empresas que publicaram o balanço social na Bovespa apresentou na média, um maior valor de mercado.

A variável de controle de tamanho da empresa confirmou um maior desempenho em 2003 das empresas da amostra, maior que o ano anterior, ainda que pequeno em todos os níveis de média $(6,34)$, mínimo $(5,43)$ e o valor máximo de $(7,98)$.

\subsection{Análise da Matriz de Correlações - 2003}

Tabela 6 - Matriz de correlações do ano de 2003

\begin{tabular}{|l|c|c|c|}
\hline \multicolumn{1}{|c|}{ Variável } & q de Tobin & Log INVTS & Log TAM \\
\hline $\log q$ de Tobin & 1,000 & & \\
\hline $\log$ INVTS & 0,046 & 1,000 & \\
\hline $\log$ TAM & 0,243 & 0,765 & 1,000 \\
\hline
\end{tabular}

As correlações diretas entre o investimento em responsabilidade social (INVESTS) e o valor da empresa ( $q$ de Tobin) se mostraram fracas estatisticamente $(0,046)$ não apresentando correlação significante estatisticamente. A variável de controle tamanho apresentou correlação significante com a variável de investimento em responsabilidade social (log INVTS) $(0,765)$.

Quanto ao sentido que as variáveis indicam:

1 A correlação encontrada $(0,046)$ entre o $q$ de Tobin e a variável (INVESTS) indica uma relação pequena entre o investimento em responsabilidade social corporativa e o valor das empresas. 
2 A correlação positiva encontrada é muito pequena para se afirmar que quanto maior o investimento em responsabilidade social, maior apresenta-se o valor de mercado da empresa.

3 Há evidências significativas de quanto maior a empresa, maior o investimento em responsabilidade Social. Isto pode significar que quando há um aumento do faturamento liquido há também um maior investimento social.

4 Contudo não se pode afirmar que houve aumento ao longo do tempo do investimento social corporativo, pois todas as demais variáveis aumentaram concomitantemente.

\subsection{Análise da Estatística Descritiva - 2004}

Tabela 07 - Estatística descritiva com valores originais 2004

\begin{tabular}{|l|c|c|c|c|c|c|}
\hline \multicolumn{1}{|c|}{$\begin{array}{c}\text { Variáveis } \\
\text { independentes }\end{array}$} & $\mathbf{N}$ & Mínimo & Média & Máximo & Assimetria & Curtose \\
\hline$q$ de Tobin & 42 & 0,343 & 1,296 & 3,209 & 0,74 & $-0,08$ \\
\hline Invests & 42 & 38793 & 553889 & 51834379 & 6,06 & 38,16 \\
\hline TAM & 42 & 324496 & 26264493 & 108201479 & 5,31 & 31,00 \\
\hline
\end{tabular}

Tabela 8 - Estatística descritiva utilizando valores em escala logarítmica 2004

\begin{tabular}{|l|c|c|c|c|c|c|}
\hline $\begin{array}{c}\text { Variáveis } \\
\text { independentes }\end{array}$ & N & Mínimo & Média & Máximo & Assimetria & Curtose \\
\hline $\log q$ de Tobin & 42 & $-0,4645$ & 0,1123 & 0,5064 & $-0,34$ & $-0,35$ \\
\hline $\log$ Invests & 42 & 4,5888 & 5,7434 & 7,7146 & 0,28 & 0,54 \\
\hline $\log$ TAM & 42 & 5,5112 & 6,4190 & 8,0342 & 0,47 & 0,51 \\
\hline
\end{tabular}

A estatística descritiva dos dados coletados para o ano de 2004 apresentou um valor médio do investimento social (log INVESTS - 5,74), maior que em 2003 (log INVESTS: 5,65). Embora ainda pequeno, revela um provável aumento na atuação social de algumas empresas ou maior transparência na divulgação dos dados do Balanço Social em relação ao ano anterior.

Igualmente ao ano 2003, observou-se que, em escala logarítmica, os dados apresentaram um padrão estatístico de assimetria e curtose mais adequado para análise estatística.

A média de valor de mercado das empresas teve também um aumento em 2004 ( $\log q$ de Tobin 0,1123) em relação a 2003 (log $q$ de Tobin 0,00503), indicando que a maioria das empresas que publicaram o balanço social na Bovespa apresentaram na média um maior valor de mercado em 2004.

A variável de controle de tamanho da empresa confirmou um aumento no desempenho das empresas em 2004, maior que o ano anterior, ainda que pequeno em todos os níveis de média $(6,41)$, mínimo $(5,51)$ e valor máximo $(8,03)$. 


\subsection{Análise da Matriz de Correlações - 2004}

Tabela 9 - Matriz de correlações do ano de 2004

\begin{tabular}{|l|c|c|c|}
\hline \multicolumn{1}{|c|}{ Variável } & q de Tobin & Log INVTS & Log TAM \\
\hline $\log q$ de Tobin & 1,000 & & \\
\hline $\log$ INVESTS & 0,168 & 1,000 & \\
\hline $\log$ TAM & 0,107 & 0,726 & 1,000 \\
\hline
\end{tabular}

1 A correlação negativa encontrada $(-0,168)$ entre o (log $q$ de Tobin) e a variável (log INVESTS) indica existência de uma relação negativa entre o investimento em responsabilidade social corporativa e o valor das empresas.

2 A correlação é muito pequena para se afirmar que quanto maior o investimento em responsabilidade social, menor o valor de mercado da empresa.

3 A correlação positiva encontrada entre o log TAM e o log INVESTS indica uma forte relação entre o tamanho da empresa e o valor do investimento em responsabilidade social corporativa, podendo-se afirmar que quanto maior a empresa, maior o investimento em responsabilidade social com base em valores explicativos da ordem de $(0,726)$. Isso pode significar que quando há um aumento do faturamento liquido há também um maior investimento social.

4 Contudo, não se pode afirmar que houve aumento ao longo do tempo do investimento social corporativo, pois todas as variáveis (tamanho $q$ de Tobin) aumentaram concomitantemente em relação aos anos anteriores.

\subsection{Análise da Regressão}

Foi realizada uma regressão que utilizou o q de Tobin como variável dependente, representando o valor de mercado das empresas e o log INVESTS como variável independente, representando o investimento social corporativo. As variáveis de controle foram representadas pelo tamanho ( $\log$ TAM); de setor de atuação foi representada pela (Dummy IND) e as Dummys: T2003 e T2002, para isolar o fator temporal.

Seguindo diversos estudos quantitativos realizados anteriormente foi usada uma transformação logarítmica das variáveis, justificada pelo fato de a transformação evitar os problemas associados a uma distribuição muito assimétrica e distante de um padrão de normalidade.

Todos os pressupostos do modelo regressivo também foram observados nos testes. Os resultados são apresentados na Tabela 10 a seguir, seguida de uma análise dos principais elementos e comportamento e relacionamento entre as variáveis. 


\subsubsection{Análise da Regressão Utilizando o log $q$ de Tobin como Variável Dependente}

Tabela 10 - Regressão Utilizando o log q Tobin como variável dependente

\begin{tabular}{|c|c|c|c|c|c|c|}
\hline $\log q$ de Tobin & Coeficiente & $\mathbf{t}$ & $P>|t|$ & R2 & $\mathbf{F}$ & $\mathbf{N}$ \\
\hline logINVESTS & 0,39 & 0,77 & 0,44 & \multirow{14}{*}{0,61} & \multirow{14}{*}{0,00} & \multirow{14}{*}{94} \\
\hline $\log$ TAM & 0,17 & 0,35 & 0,72 & & & \\
\hline d1 (alimentos/beb ) & $-0,81$ & $-2,32$ & 0,02 & & & \\
\hline d2 (energia elétrica) & $-0,30$ & $-1,57$ & 0,12 & & & \\
\hline d3 (outros) & $-0,77$ & $-3,29$ & 0,00 & & & \\
\hline d4 (papel e celulose) & $-0,22$ & $-1,09$ & 0,28 & & & \\
\hline d5 (petróleo e gás) & $-0,39$ & $-1,72$ & $-0,09$ & & & \\
\hline d6 (química) & $-0,212$ & $-1,01$ & 0,32 & & & \\
\hline d7 (siderurgia ..) & $-0,38$ & $-1,79$ & 0,78 & & & \\
\hline d8 (telecomunicações) & $-0,35$ & $-1,61$ & 0,11 & & & \\
\hline d9 (têxtil...) & $-0,33$ & $-1,73$ & 0,09 & & & \\
\hline d10 (veículos e peças) & $-0,60$ & $-2,60$ & 0,01 & & & \\
\hline T2003 & $-0,04$ & $-1,24$ & 0,22 & & & \\
\hline Cons & $-2,49$ & $-0,87$ & 0,39 & & & \\
\hline
\end{tabular}

Os resultados alcançados pela regressão do período ratificaram os resultados encontrados nas matrizes de correlação dos anos de 2002, 2003, 2004 em relação à variável dependente $q$ de Tobin, que, representando o valor de mercado das empresas, não apresentou relação estatisticamente significante com a variável independente INVESTS (investimento social corporativo). O valor de teste $F(0,00000)$ foi significante ao nível de $5 \%$ e o R2 de 0.6176 refletiu considerável poder explicativo do modelo.

Da mesma forma, não se pode afirmar a existência de uma relação entre o $q$ de Tobin e o tamanho das empresas com investimentos sociais corporativos, uma vez que resultados alcançados pela regressão não apresentaram relação estatisticamente significante.

Em relação ao setor, a variável dos setores de veículo e peças, alimentos e bebidas apresentaram relação significativa ao nível de 5\%, e os setores petróleo e gás, siderurgia e têxtil apresentaram relação significativa ao nível de 10\%. Os demais setores não tiveram relação estatística significativa. O fato a ser destacado é que em todos os setores cuja relação foi significativa constatou-se a presença de relação negativa entre o investimento social e o valor das empresas.

Com objetivo de melhor observação dos resultados obtidos na regressão nos períodos analisados, segue abaixo um quadro-resumo com os principais resultados observados na regressão. 


\subsubsection{Resumo dos Resultados Obtidos na Regressão}

Quadro 3 - Resumo do Resultado das Regressões

\begin{tabular}{|c|l|l|l|}
\hline Hipótese 1 & Hipótese 2 & \multicolumn{1}{|c|}{ Hipótese 3 } & \multicolumn{1}{c|}{ Hipótese 4 } \\
\hline $\begin{array}{c}\text { Relação não } \\
\text { Confirmada }\end{array}$ & $\begin{array}{l}\text { Relação não } \\
\text { Confirmada }\end{array}$ & $\begin{array}{l}\text { Relação não } \\
\text { confirmada }\end{array}$ & $\begin{array}{l}\text {-Rejeitada a hipótese } \\
\text { para os 5 setores: } \\
\text { veículos e peças, } \\
\text { alimentos e bebidas, } \\
\text { petróleo e gás, } \\
\text { siderurgia e têxtil; } \\
\text {-Não confirmada } \\
\text { relação para os } \\
\text { demais setores. }\end{array}$ \\
\hline
\end{tabular}

\subsection{Análise Crítica dos Resultados}

Após a investigação da literatura, formularam-se duas hipóteses centrais e duas hipóteses complementares, conforme já explicitado anteriormente. Utilizando-se ferramentas como a estatística descritiva, a matriz de correlação e a regressão múltipla, concluiu-se, pelos resultados, que a primeira hipótese principal não pôde ser confirmada, uma vez que a relação encontrada não apresentou significância estatística ao nível de 5\%.

Essa ausência de relação significante entre as variáveis de valor de mercado e investimento social corporativo confirma os resultados da pesquisa de Borba (2005, p. 11), que concluiu: "[...] utilizando indicadores de desempenho financeiro-contábeis ou de mercado, a relação nula foi preponderante, ou seja, foi constatada a ausência de relação significante entre as variáveis de desempenho social e financeiro corporativos utilizados".

A segunda hipótese central não foi confirmada, uma vez que os resultados de existência de relação positiva entre o investimento social corporativo e o valor das empresas não foi significativo na regressão e nem nas matrizes de correlação dos anos de 2002, 2003 e 2004, não se confirmando os argumentos de Preston e O`Bannon (1997) de existência de relação positiva.

Diante desses resultados, não é possível confirmar os argumentos de existência de ganhos obtidos pelas empresas por assumirem práticas socialmente responsáveis; e que o investimento social corporativo causa impacto positivo na avaliação da empresa no mercado, conforme defendido por Ashley et al. (2005).

Pelo sentido da direção negativa apresentada nos coeficientes da regressão e pela matriz de correlações nos anos de 2002 e 2004, não se pode aceitar ou rejeitar o argumento de que um maior investimento social corporativo acarretaria em uma piora no valor de mercado da empresa. Na visão de Friedman (1970), essa depreciação no valor da empresa se justificaria em razão da realização de gastos em atividade não relacionados diretamente com o negócio principal. 
Quanto à hipótese complementar de que existe relação positiva entre o valor das empresas com investimento social corporativo e tamanho, os testes não confirmaram haver uma relação significativa ao nível de $5 \%$. Porém, a correlação positiva encontrada entre o log TAM e o log INVESTS indica uma relação entre o tamanho da empresa e o valor do investimento em responsabilidade social corporativa, indicando que quanto maior o tamanho da empresa, maior o investimento em responsabilidade. Isso pode significar que empresas com maior faturamento líquido também apresentam maior investimento social corporativo.

Em relação à hipótese complementar de que existe uma relação positiva entre o valor das empresas com investimento social corporativo e o setor onde a empresa atua, a hipótese foi rejeitada, pois, para alguns setores, essa relação foi confirmada com o sentido de relação negativa e, para outros setores, não pôde ser rejeitada por não ser significativa estatisticamente. Esses resultados confirmam o que para Griffin e Mahon (1997) é essencial: a necessidade em diferenciar os setores em relação às suas especificidades sociais.

Os resultados encontrados oferecem também embasamento para não rejeição dos argumentos da abordagem econômica das externalidades em relação ao investimento social, cuja proposição de formas para corrigir falhas de mercado seria a internalização dos custos produzidos pela externalidade negativa, conforme salientam Pindyck e Rubinfeld (2002). Essa produção de externalidades com origens teóricas na economia clássica poderia ser uma alternativa explicativa para os resultados encontrados na análise da relação entre o valor do investimento social corporativo e o valor de mercado das empresas analisadas, uma vez que, ao apresentar resultados não significativos de relacionamento, pode significar que o esforço financeiro feito por essas corporações na área socioeconômica (embora justificada pelo impacto que as atividades principais dessas corporações ocasionam aos seus diversos stakeholders) seja absorvido totalmente pelo processo produtivo, e que seriam insignificantes quando inclusos no custo marginal dos produtos, para sustentar qualquer tipo de relação com o valor de mercado dessas corporações quando analisadas como um todo.

Entretanto, quando essa análise ocorre por setor de atividade, percebe-se que alguns setores mencionados anteriormente apresentaram relação negativa significativa entre o valor do investimento social corporativo e o valor de mercado das empresas nesses setores, ou seja, quanto maior o valor de mercado, menor o investimento social corporativo e vice-versa. Isso pode indicar que em tais setores a produção de externalidades afeta negativamente os resultados corporativos.

$\mathrm{Na}$ busca de responder ao problema de pesquisa e ao objetivo geral, concluiu-se por meio de análise estatística que a relação entre o investimento social corporativo e o valor das empresas brasileiras com ações negociadas na Bovespa que publicam regularmente o balanço social no Ibase não pôde ser confirmada, uma vez que os resultados não foram significantes estatisticamente. 


\section{CONCLUSÃO}

Os resultados encontrados por esta pesquisa não evidenciaram que uma administração voltada para os interesses de todos os envolvidos acarretaria um melhor ou um pior desempenho no valor de mercado das empresas.

Encontrou-se relação não significativa entre a variável de controle tamanho da empresa e o q de Tobin em empresas com investimentos sociais corporativos. Isso pode indicar que o valor de mercado das empresas brasileiras com maior porte não se diferencia das demais empresas que adotam práticas sociais corporativas.

Entretanto, a pesquisa confirmou a necessidade em diferenciar os setores em relação às suas práticas sociais. Investigou-se no total dez setores - alimentos e bebidas; energia elétrica; papel e celulose; petróleo e gás; química; siderurgia; telecomunicações; têxtil; veículos e peças, além de outros. Dos setores pesquisados, constatou-se a presença de resultado estatisticamente significante no sentido da relação negativa para cinco setores da economia (veículo e peças; alimentos e bebidas; petróleo e gás; siderurgia e têxtil), ou seja, quanto mais essas empresas investiram em práticas socialmente responsáveis, maior foi o decréscimo no seu valor de mercado.

Apesar de ser um tema ainda recente no campo da Contabilidade e da Administração no Brasil, a relação entre o investimento social privado e o valor de mercado das empresas listadas na Bovespa é um tema cujo debate continua em aberto, ainda sem conclusões definitivas.

Como principais limitações da pesquisa, identificaram-se o tamanho da amostra e a utilização apenas de dados secundários. Para pesquisas futuras, recomenda-se a utilização de uma amostra mais robusta e de dados primários, como alternativa metodológica para confirmação dos resultados.

\section{REFERÊNCIAS}

ASHLEY, P. A. et al. Ética e responsabilidade social nos negócios. São Paulo: Saraiva, 2002.

,Ética e responsabilidade social nos negócios. 2. ed. São Paulo: Saraiva, 2005.

BORBA, P. da R. F. Relação entre desempenho social corporativo e desempenho financeiro de empresas no Brasil. 2005. Dissertação (Mestrado em Administração) - Faculdade de Economia, Administração e Contabilidade (FEA) Universidade de São Paulo, São Paulo, 2005.

BROMILEY, P.; MARCUS, A. 1989. The deterrent to duious corporate behavior: profitability, probability and safety recalls. Strategic Management Journal, v. 10: 233-50. 
CARROLL, A. B. A three-dimensional conceptual model of corporate performance. Academy of Management Review, v. 4, p. 497-505, 1979.

. The Pyramid of corporate social responsibility: toward the moral management of organizational stakeholders. Business Horizons, v. 34, n. 4, p. 39-48, jul./aug. 1991.

1999.

. Corporate social responsibility. Business and Society, v. 8, n.3, p. 268-295, sep.

CORNELL, B. \& SHAPIRO, A. Corporate stakeholders and corporate finance. Financial Management, 16:5-14,

DONALDSON, T.; PRESTON, L. E. The stakeholder theory of the corporation:concepts, evidence and implications. Academy of Management Review. Ada, v. 20, n. 1, p. 65-91, 1995.

FAMÁ, R.; BARROS, L.B.C. Q de Tobin e seu uso em finanças: aspectos metodológicos e Conceituais. Caderno de pesquisas em administração, São Paulo, v.07, nº 4, out/dez 2000.

FERREL, O.C; FRAEDRICH, J; FERRELL, L. Ética empresarial: dilemas, tomadas de decisões e casos ( tradução) Rio de Janeiro: Reichmann \& Affonso, 2001.

FREEMAN, T. E. Strategic management: a stakeholder approach. Boston: Pitman, 1984.

FRIEDMAN, M. The social responsibility of business is to increase its profits. New York Times Magazine, 13 Sept. 1970.

GRIFFIN, J. J.; MAHON, J. F. The corporate social performance and corporate financial performance debate: twenty-years of incomparable research. Business and Society. Chicago, v. 36, n. 1, p. 5-31, mar. 1997.

Ibase - INSTITUTO BRASILEIRO DE ANÁLISES SOCIAIS E ECONÔMICAS, 2006. Balanço Social. Disponível em: http://www.balancosocial.org.br/. Acesso em: 20 mai. 2005.

MCGUIRE, J. B., SUNDGREN, A.; Schneeweis, T. 1988. Corporate social responsibility and firm financial performance. Academy of Management Journal, 31(4): 854-872.

PRESTON, L. E.; O'BANNON, D. P. The corporate social-financial relationship: typology and analysis. Business and Society, v. 36, n. 4, p. 419-420, 1997.

PINDYCK, R. S.; RUBINFELD, D. L. Microeconomia. 5 ed. São Paulo: Makron Books, 1999.

RH Coase, 3 Journal of Law and Economics 1-44 'The Problem of Social Cost' (1960)

Ruf, B.; Muralidhar, K.; Brown, R.; Janney, J. \& Paul, K. (2001). An Empirical Investigati on of the Relationship between Change in Corporate Social Performance and Financial Performance: A Stakeholder Theory Perspective. Journal of Business Ethics, 32 (2), 143-156.

TRIPODI, T. et al. Análise da pesquisa social. Rio de Janeiro: Francisco Alves, 1975. 
WOOD, D. J. Corporate social performance revisited. Academy of Management Review. v. 16, n. 4, p. 691-718, oct. 1991.

WOOD, D. J.; JONES R. E. Research incorporate social performance: what have we learned? In: BURLINGAME, D. F.; YOUNG, D. R. ( Eds). Corporate philanthropy at the crossroads. Bloomington, Indiana: University Press, 1996.

ULLMANN, A. A. Data in search of a theory: a critical examination of the relationships among social performance, social disclosure, and economic performance of U.S. firms. The Academy of Management Review, Vol. 10, nº 3 (Jul., 1985), pp. 540-557. 